\title{
BUCKLING, BARRELLING, AND SURFACE INSTABILITIES OF A FINITE, TRANSVERSELY ISOTROPIC CIRCULAR CYLINDER
}

\author{
BY
}

K. T. CHAU

The Hong Kong Polytechnic University, Hung Hom, Hong Kong

\begin{abstract}
This paper investigates all possible geometric instabilities, such as buckling, barrelling, surface wrinkling, and orange-peel mode instabilities, for a finite length transversely isotropic circular cylinder under axisymmetric tension or compression. The constitutive responses of the cylinder can, in general, be transversely isotropic, compressible, and elastic-plastic; and no existence of rate potential is assumed. A general procedure is given to calculate the smallest critical ratio of radius to length, $(a / L)_{\mathrm{cr}}$, of a circular cylinder that separates the buckling (antisymmetric) and the barrelling (axisymmetric) instabilities. Numerical results show that $(a / L)_{\mathrm{cr}}$ is very sensitive to the constitutive model used. The stress at the maximum load point is obtained as the first term of the axisymmetric long wavelength limit; the Euler buckling formula is obtained as the first term of the antisymmetric long wavelength limit. Three different types of surface instabilities are considered. The eigenstress for the longitudinal short wavelength limit corresponds to that for the wrinkling instability of a halfspace under plane deformations; the circumferential short wavelength limit is always possible; and the longitudinal and circumferential short wavelength limit, which corresponds to the surface instability with an "orange-peel" appearance, is available along the tensile elliptic-parabolic boundary. Numerical results show that eigenmodes of $n \geq 2$ (where $n$ is the circumferential wave number) can be the first bifurcation encountered under tension depending on the geometric ratio, $a / L$. However, such eigenmodes will not be the first possible bifurcation under compression unless the ratio of the incremental transverse shear modulus to the incremental longitudinal shear modulus drops below, approximately, 0.25 .
\end{abstract}

1. Introduction. It is well known that localization of deformation in solids can be understood as a result of material instability (Rudnicki and Rice [21]; Rice [17]). In particular, the bifurcation analysis by Rudnicki and Rice [21] suggests that strain localization in rocks under axisymmetric compression appears relatively late in the

Received March 3, 1992.

1991 Mathematics Subject Classification. Primary 73H99; Secondary 73B40, 35 Q72.

(C)1995 Brown University 
post-peak applied stress regime; however, Santarelli and Brown [22] reported that localization is often observed preceding the peak applied stress.

Recently, Chau [7] argued that geometric diffuse modes, such as bulging, necking, and surface rumpling, may trigger nonhomogeneous deformation and, eventually, lead to pre-peak localization of deformation reported by Santarelli and Brown [22]. In order to further understand this possibility, Chau [8] examined the antisymmetric diffuse modes, such as buckling, of a finite circular cylinder under axisymmetric tension and compression. However, so far only the axisymmetric (Chau [7]) and the antisymmetric (Chau [8]) diffuse bifurcation modes have been investigated.

In this study, we consider a bifurcation analysis to obtain all the possible diffuse mode bifurcations for a finite circular cylinder which is characterized by the most general transversely isotropic model. The special cases of the general results of this analysis include both the axisymmetric and the antisymmetric solutions obtained previously by Chau $[7,8]$.

In an attempt to predict whether buckling or barrelling occurs first under compression, Davies [11] compared the bifurcation conditions for the barrelling and the buckling instabilities of a finite cylinder. Davies' work [11] is a continuation of the theoretical works obtained by Simpson and Spector [24, 25], and by Davies [10]. However, due to mathematical difficulties, Davies [11] compared only the critical stretch for the barrelling of a circular cylinder to that for the buckling of a square cylinder. The present bifurcation analysis may be used to predict whether the first bifurcation of a finite circular cylinder under compression is buckling or barrelling. The experimental observations by Beatty [3] for rubber seem to suggest that there is a critical geometrical ratio that separates the barrelling bifurcation from the buckling bifurcation for a cylinder. However, this conjecture has not been proved conclusively. Furthermore, Levinson [15] and Davies [10] argued that Beatty's experimental results may be due to end friction appearing between the end of the cylinder and the testing apparatus. Nevertheless, we attempt, in this study, to obtain such a critical geometric ratio by solving the axisymmetric and the antisymmetric eigenvalue equations simultaneously.

Another motivation of the present analysis is to provide the bifurcation conditions for surface instabilities, including the parallel wrinkle mode and the "orange-peel" mode, of a circular cylinder observed experimentally by Rittel et al. [19] under tension and by Rittel [18] under compression. Such surface instabilities of a circular cylinder are obtained from the short wavelength limits of the general eigenvalue equation. Three different short wavelength limits are considered: (1) the longitudinal short wavelength limit; (2) the circumferential short wavelength limit; and (3) the longitudinal and circumferential short wavelength limit.

2. Mathematical formulation. We consider a circular cylinder of radius $a$ and length $L$ under initial axisymmetric deformations. The current configuration is adopted as reference and the subsequent deformation of the material is characterized by the following time- and rate-independent incrementally linear solid: 


$$
\begin{gathered}
\stackrel{\nabla}{\sigma}_{r r}=C_{11} D_{r r}+C_{12} D_{\theta \theta}+C_{13} D_{z z}, \quad \stackrel{\nabla}{\sigma}_{\theta \theta}=C_{12} D_{r r}+C_{11} D_{\theta \theta}+C_{13} D_{z z}, \\
\stackrel{\nabla}{\sigma}_{z z}=C_{31}\left(D_{r r}+D_{\theta \theta}\right)+C_{33} D_{z z}, \quad \stackrel{\nabla}{\sigma \theta}_{r \theta}=\left(C_{11}-C_{12}\right) D_{r \theta}, \\
\stackrel{\nabla}{\sigma \alpha}_{z \alpha}=2 C_{44} D_{z \alpha} \quad(\alpha=r, \theta)
\end{gathered}
$$

where $(r, \theta, z)$ are the cylindrical polar coordinates, $\sigma$ is the Cauchy stress tensor, the superposed $\nabla$ denotes the Jaumann or co-rotational rate (Prager [16]), and D is the rate of deformation tensor.

The constitutive law, (2.1), is motivated by its possible application to frictional and dilatant (i.e., compressible) solids, such as brittle rocks. In general, the constitutive response of the materials described by (2.1) can be transversely isotropic, compressible, and elastic-plastic. Furthermore, plastic normality (or associate flow rule) does not hold for this class of materials since $C_{13} \neq C_{31}$. An important consequence of this "non-normality" in (2.1) is that the variational structure of the governing incremental field equations is lost, that is, rate potential does not exist in the solids. Consequently, as discussed by Chau and Rudnicki [9] and by Chau [7], Hill's [12-14] theory of bifurcation and uniqueness cannot be applied. Furthermore, note that the incremental material parameters in (2.1) can, in general, change with the varying applied stress. That is, the material described by $(2.1)$ is only incrementally linear.

The number of incremental constitutive parameters may be reduced to five if the constitutive response of the transversely isotropic elastic-plastic solids admits a rate potential, that is, $C_{13}=C_{31}$. Rudnicki's model [20], for pressure-sensitive dilatant materials, can be recovered as a special case of (2.1) by using the following identifications:

$$
\begin{array}{ccc}
C_{11}=G_{t}+9 K / 4, & C_{12}=9 K / 4-G_{t}, & C_{13}=9 K \nu / 2, \\
C_{31}=9 K r^{*} / 4, & C_{33}=E+9 K r^{*} \nu / 2, & C_{44}=G_{l},
\end{array}
$$

where $E, G_{l}, G_{t}, K, r^{*}$, and $\nu$ are the incremental Young's modulus, the longitudinal shear modulus, the transverse shear modulus, the in-plane bulk modulus, the pressure-sensitive factor, and the effective Poisson's ratio, respectively. For the physical meanings of these parameters, we refer to Rudnicki [20] or Chau [7]; they also discuss the limitations of the constitutive models having the form of (2.1).

It will be helpful to use the definitions of the instantaneous tangent modulus $\left(E_{\tan }\right)$ and the stress at maximum load point $\left(\sigma_{z z}^{\max }\right)$ in later discussions for the axisymmetric and antisymmetric long wavelength limits. Hence, we will derive $E_{\tan }$ and $\sigma_{z z}^{\max }$ next before our discussion on the equilibrium equations and the boundary conditions for the incremental velocity field.

If we set the Jaumann rates of $\sigma_{r r}$ and $\sigma_{\theta \theta}$ to zero in (2.1), the following instantaneous tangent modulus relating the Jaumann rate of $\sigma_{z z}$ and $D_{z z}$ is obtained:

$$
E_{\tan }=\frac{C_{33}\left(C_{12}+C_{11}\right)-2 C_{31} C_{13}}{C_{11}+C_{12}}
$$

Under an axisymmetric homogeneous state with nonzero axial stress $\sigma_{z z}$ and constant lateral stresses $\sigma_{r r}=\sigma_{\theta \theta}$, the condition for the maximum load point is (Chau 
[7]):

$$
\dot{t}_{z z}=\dot{t}_{\theta \theta}=\dot{t}_{r r}=0,
$$

(where $\dot{t}$ is the nominal stress rate tensor). This condition yields three equations for $D_{r r}, D_{\theta \theta}$, and $D_{z z}$. A nontrivial solution for $\mathbf{D}$ yields the stress at maximum load point as

$$
\sigma_{z z}^{\max }=\frac{C_{33}\left(C_{12}+C_{11}\right)-2 C_{31} C_{13}+\sigma_{r r}\left(C_{33}-2 C_{31}\right)}{2\left(C_{13}+\sigma_{r r}\right)} .
$$

Note that the instantaneous tangential modulus equals the stress at maximum load point (i.e., $E_{\tan }=\sigma_{z z}^{\max }$ ) only if $\sigma_{r r}=0$ and $2 C_{13}=C_{11}+C_{12}$. The requirement corresponds to $\nu=1 / 2$ in terms of Rudnicki's model [20] and this is consistent with the observation by Chau and Rudnicki [9]. More specifically, $E_{\tan }=\sigma_{z z}^{\max }$ is true only for incompressible materials.

A cylindrical polar coordinate system $(r, \theta, z)$ with the origin resting on the bottom of the cylinder and with the axis of symmetry as the $z$-axis is used. The ends $(z=0, L)$ are loaded by a prescribed uniform velocity field in the $z$-direction with no shear traction; and a constant lateral stress is applied in the $r$-direction with no shear traction on the curved boundary $(r=a)$. We assume that the circular cylinder deforms homogeneously up to the current state and further homogeneous deformation is always possible for the next increment of deformation. We look for an alternative inhomogeneous solution that satisfies the continued equilibrium and the boundary conditions.

Note that, for hyperelastic solids, the existence of an initial homogeneous state is only guaranteed for materials satisfying some particular constitutive hypotheses; for example, see Sec. 3 of Simpson and Spector [24]. However, for the class of material considered here, we do not attempt to prove the existence of this homogeneous state of deformation.

Rewriting (2.1) in terms of the rate of nominal stress and substituting the result into the equilibrium rate equations, $\boldsymbol{\nabla} \cdot \dot{\mathbf{t}}^{*}=0$, yields

$$
\begin{gathered}
C_{11} \frac{\partial e}{\partial r}+\left(C_{11}-C_{12}\right) \frac{1}{r} \frac{\partial \Omega_{r \theta}}{\partial \theta}+C_{44}(1+s) \frac{\partial^{2} \nu_{r}^{*}}{\partial z^{2}}+\left[C_{13}-C_{11}+C_{44}(1-s)\right] \frac{\partial^{2} v_{z}^{*}}{\partial r \partial z}=0, \\
C_{11} \frac{1}{r} \frac{\partial e}{\partial \theta}+\left(C_{11}-C_{12}\right) \frac{\partial \Omega_{\theta r}}{\partial r}+C_{44}(1+s) \frac{\partial^{2} v_{\theta}^{*}}{\partial z^{2}}+\left[C_{13}-C_{11}+C_{44}(1-s)\right] \frac{1}{r} \frac{\partial^{2} v_{z}^{*}}{\partial \theta \partial z}=0, \\
\left(C_{31}+2 C_{44}\right) \frac{\partial e}{\partial z}+\left[C_{33}-C_{31}-2 C_{44}\right] \frac{\partial^{2} v_{z}^{*}}{\partial z^{2}}+C_{44}(1-s)\left[\frac{1}{r} \frac{\partial \Omega_{z \theta}}{\partial \theta}+\frac{1}{r} \frac{\partial}{\partial r}\left(r \Omega_{z r}\right)\right]=0,
\end{gathered}
$$

where

$$
s=\left(\sigma_{z z}-\sigma_{r r}\right) /\left(2 C_{44}\right), \quad e=\frac{1}{r} \frac{\partial\left(r v_{r}^{*}\right)}{\partial r}+\frac{1}{r} \frac{\partial v_{\theta}^{*}}{\partial \theta}+\frac{\partial v_{z}^{*}}{\partial z},
$$

and $\Omega=\left(\mathbf{L}-\mathbf{L}^{\mathrm{T}}\right) / 2$ is the antisymmetric part of the velocity gradient tensor. The superscript ${ }^{*}$ denotes the difference between the nonuniform and the homogeneous solutions. 
The boundary conditions governing the difference in the incremental solutions are

$$
v_{z}^{*}=0, \quad \dot{i}_{z r}^{*}=\left(C_{44}-\sigma_{r r}\right) \frac{\partial v_{z}^{*}}{\partial r}+C_{44} \frac{\partial v_{z}^{*}}{\partial z}=0, \quad i_{z \theta}^{*}=\left(C_{44}-\sigma_{r r}\right) \frac{1}{r} \frac{\partial v_{z}^{*}}{\partial \theta}+C_{44} \frac{\partial v_{\theta}^{*}}{\partial z}=0
$$$$
\text { on } z=0, L ; \text { and }
$$

$$
\begin{aligned}
& \dot{t}_{r r}^{*}=C_{11} \frac{\partial v_{r}^{*}}{\partial r}+\left(C_{12}+\sigma_{r r}\right)\left[\frac{v_{r}^{*}}{r}+\frac{1}{r} \frac{\partial v_{\theta}^{*}}{\partial \theta}\right]+\left(C_{13}+\sigma_{r r}\right) \frac{\partial v_{z}^{*}}{\partial z}=0, \\
& \dot{t}_{r \theta}^{*}=\left[\frac{1}{2}\left(C_{11}-C_{12}\right)-\sigma_{r r}\right]\left(\frac{1}{r} \frac{\partial v_{r}^{*}}{\partial \theta}-\frac{v_{\theta}^{*}}{r}\right)+\frac{1}{2}\left(C_{11}-C_{12}\right) \frac{\partial v_{\theta}^{*}}{\partial r}=0, \\
& \dot{t}_{r z}^{*}=\left(C_{44}-\sigma_{z z}\right) \frac{\partial v_{r}^{*}}{\partial z}+C_{44} \frac{\partial v_{z}^{*}}{\partial r}=0,
\end{aligned}
$$

on $r=a$. Note that boundary conditions are written in terms of the incremental velocity field $\mathbf{v}^{*}$, the constitutive tensor $\mathbf{C}$, and the current stress state. Then the bifurcation analysis requires solving the three coupled partial differential equations (2.6) and boundary conditions (2.8) and (2.9) for $\mathbf{v}^{*}$.

3. Method of solution. The method of solution for the continued equilibrium equations for a transversely isotropic solid, characterized by Rudnicki's model [20], has been discussed by Chau [8]. The method is generalized here to the bifurcation analysis for the most general transversely isotropic solids described by (2.1). Two velocity potentials $\Phi$ and $\Psi$ are introduced such that

$$
\begin{aligned}
& v_{r}^{*}=\frac{\partial^{2} \Phi}{\partial r \partial z}+\frac{1}{r} \frac{\partial \Psi}{\partial \theta}, \quad v_{\theta}^{*}=\frac{1}{r} \frac{\partial^{2} \Phi}{\partial \theta \partial z}-\frac{\partial \Psi}{\partial r}, \\
& v_{z}^{*}=-\left[\frac{C_{11}}{C_{13}+C_{44}(1-s)}\right] \nabla_{1} \Phi-\left[\frac{C_{44}(1+s)}{C_{13}+C_{44}(1-s)}\right] \frac{\partial^{2} \Phi}{\partial z^{2}},
\end{aligned}
$$

where

$$
\nabla_{1}=\frac{1}{r} \frac{\partial}{\partial r}\left(r \frac{\partial}{\partial r}\right)+\frac{1}{r^{2}} \frac{\partial^{2}}{\partial \theta^{2}}
$$

In order to satisfy the equilibrium equations, (2.6), we have the following governing equations for $\Phi$ and $\Psi$ :

$$
\left(\nabla_{1}-\nu_{1}^{2} \frac{\partial^{2}}{\partial z^{2}}\right)\left(\nabla_{1}-\nu_{2}^{2} \frac{\partial^{2}}{\partial z^{2}}\right) \Phi=0, \quad\left(\nabla_{1}+\nu_{3}^{2} \frac{\partial^{2}}{\partial z^{2}}\right) \Psi=0,
$$

and $\nu_{1}$ and $\nu_{2}$ satisfy

$$
A \nu_{\alpha}^{4}+B \nu_{\alpha}^{2}+C=0 \quad(\alpha=1,2),
$$

where $A, B$, and $C$ are defined as

$$
\begin{gathered}
A=C_{11} C_{44}(1-s), \quad B=C_{11} C_{33}-C_{13} C_{31}-C_{44}\left(C_{13}+C_{31}\right)+C_{44}\left(C_{31}-C_{13}\right) s, \\
C=C_{33} C_{44}(1+s),
\end{gathered}
$$

and $\nu_{3}$ is defined as

$$
\nu_{3}=\left[2 C_{44}(1+s) /\left(C_{11}-C_{12}\right)\right]^{1 / 2} .
$$


As remarked by Chau [8], there exists a close resemblance between (3.4) and the characteristic shear band equation. More specifically, $\nu_{\alpha}$ satisfies the same characteristic equation for the normal to the shear band (see Chau $[7,8]$ ). The solutions of (3.3) can, therefore, be classified into three different regimes: elliptic (consisting of the elliptic imaginary and the elliptic complex subregimes), hyperbolic, or parabolic. The classification of regimes for Rudnicki's model [20] has been given by Chau [7]. Briefly, the different regimes are

(1) Elliptic Imaginary: $B^{2}-4 A C>0 ; A C>0 ; B>0$;

(2) Elliptic Complex: $B^{2}-4 A C<0 ; A C>0$;

(3) Hyperbolic: $B^{2}-4 A C>0 ; A C>0 ; B<0$; and

(4) Parabolic: $A C<0$, or simply $|s|>1$.

The boundary conditions, (2.9), become (in terms of $\Phi$ and $\Psi$ )

$$
\begin{gathered}
\left(Y_{1} \nabla_{1}+Y_{2} \frac{\partial^{2}}{\partial z^{2}}\right) \frac{\partial \Phi}{\partial z}+Y_{3}\left[\frac{\partial^{3} \Phi}{\partial z \partial r^{2}}+\frac{\partial}{\partial r}\left(\frac{1}{r} \frac{\partial \Psi}{\partial \theta}\right)\right]=0 \\
\left(Y_{4} \frac{\partial}{\partial r} \nabla_{1}+Y_{5} \frac{\partial^{3}}{\partial r \partial z^{2}}\right) \Phi+Y_{6} \frac{1}{r} \frac{\partial^{2} \Psi}{\partial \theta \partial z}=0 \\
Y_{3} \frac{\partial}{\partial r}\left(\frac{1}{r} \frac{\partial^{2} \Phi}{\partial \theta \partial z}\right)+Y_{7}\left(\frac{1}{r} \frac{\partial \Psi}{\partial r}+\frac{1}{r^{2}} \frac{\partial^{2} \Psi}{\partial \theta^{2}}\right)-Y_{8} \frac{\partial^{2} \Psi}{\partial r^{2}}=0
\end{gathered}
$$

on $r=a$; and, where

$$
\begin{aligned}
& Y_{1}=C_{12}+\sigma_{r r}-\frac{C_{11}\left(C_{13}+\sigma_{r r}\right)}{C_{13}+C_{44}(1-s)}, \quad Y_{2}=-\frac{C_{44}\left(C_{13}+\sigma_{r r}\right)(1+s)}{C_{13}+C_{44}(1-s)}, \\
& Y_{3}=C_{11}-C_{12}-\sigma_{r r}, \quad Y_{4}=-\frac{C_{11} C_{44}(1-s)}{C_{13}+C_{44}(1-s)}, \\
& Y_{5}=\frac{C_{44}(1-s)\left(C_{13}-2 C_{44} s\right)}{C_{13}+C_{44}(1-s)}-\sigma_{r r}, \quad Y_{6}=C_{44}(1-s)-\sigma_{r r}, \\
& Y_{7}=\frac{1}{2}\left(C_{11}-C_{12}-2 \sigma_{r r}\right), \quad Y_{8}=\frac{1}{2}\left(C_{11}-C_{12}\right) .
\end{aligned}
$$

4. Diffuse bifurcation modes. We consider the following eigenmodes for the velocity potentials:

$$
\Phi=\varphi(r) \sin (\eta z) \cos (n \theta), \quad \Psi=\psi(r) \cos (\eta z) \sin (n \theta),
$$

where $\eta=m \pi / L$, with $m, n=1,2, \ldots$. Note that the axisymmetric modes correspond to $n=0$; and the antisymmetric modes correspond to $n=1$. The end boundary condition, (2.8), is satisfied exactly by the bifurcation modes (4.1). Substitution of (4.1) into (3.3) gives

$$
\left(\nabla_{2}+\eta^{2} \nu_{1}^{2}\right)\left(\nabla_{2}+\eta^{2} \nu_{2}^{2}\right) \varphi=0, \quad\left(\nabla_{2}-\eta^{2} \nu_{3}^{2}\right) \psi=0
$$

where

$$
\nabla_{2}=\frac{1}{r} \frac{d}{d r}\left(r \frac{d}{d r}\right)-\frac{n_{2}}{r^{2}}
$$


The general solutions for $\varphi$ and $\psi$ are the Bessel function and the modified Bessel function of order $n$, respectively. Thus, the general solution for $\varphi$ is

$$
\varphi=A_{1} J_{n}\left(\eta \nu_{1} r\right)+A_{2} J_{n}\left(\eta \nu_{2} r\right),
$$

where $J_{n}(x)$ is the Bessel function of order $n$, and the constants $A_{1}$ and $A_{2}$ are, in general, complex. Substitution of (4.4) into the first of (4.2) leads to (3.4) again; hence, the classification of the parameter regime remarked earlier also applies here. For $\nu_{3}^{2}>0$, the general solution form for $\psi$ is

$$
\psi=A_{3} I_{n}\left(\eta \nu_{3} r\right)
$$

where $I_{n}(x)$ is the modified Bessel function of order $n$, and $A_{3}$ is a real constant. As expected, substitution of (4.5) into the second of (4.2) gives (3.6) again.

Thus, the boundary conditions, (2.9), in terms of $\varphi$ and $\psi$, become

$$
\begin{gathered}
\eta\left(Y_{1} \nabla_{2}-\eta^{2} Y_{2}\right) \varphi+Y_{3}\left[\eta \frac{d^{2} \varphi}{d r^{2}}+n \frac{d}{d r}\left(\frac{\psi}{r}\right)\right]=0, \\
\left(Y_{4} \frac{d}{d r} \nabla_{2}-\eta^{2} Y_{5} \frac{d}{d r}\right) \varphi-Y_{6} \eta n \frac{\psi}{r}=0, \\
-\eta n Y_{3} \frac{d}{d r}\left(\frac{\varphi}{r}\right)+Y_{7}\left(\frac{1}{r} \frac{d \psi}{d r}-\frac{n^{2}}{r^{2}} \psi\right)-Y_{8} \frac{d^{2} \psi}{d r^{2}}=0,
\end{gathered}
$$

on $r=a$, where the $Y_{i}, i=1, \ldots, 8$, are given in (3.8) and $\nabla_{2}$ is defined in (4.3). Since the calculation for the eigenvalue equations is similar to that for the axisymmetric diffuse modes considered by Chau [7], only the results are summarized briefly. The long wavelength limit and the short wavelength limits will be considered separately in later sections.

As mentioned earlier, the elliptic regime can further be subdivided into the elliptic complex (EC) subregime, where the roots for (3.4) are complex conjugate pairs, and the elliptic imaginary (EI) subregime, where the roots for (3.4) are pure imaginary. In the elliptic complex subregime, the solution forms for $\varphi$ and $\psi$ are

$$
\varphi=A_{1} J_{n}(\eta \nu r)+\bar{A}_{1} J_{n}(\eta \bar{\nu} r), \quad \psi=A_{3} I_{n}\left(\eta \nu_{3} r\right),
$$

where the superposed bar denotes the complex conjugate. The complex conjugate pairs of $\nu_{\alpha}$ are $\nu=p+i q$ and $\bar{\nu}=p-i q ; p$ and $q$ are defined as

$$
p^{2}-q^{2}=-B /(2 A), \quad p^{2}+q^{2}=(C / A)^{1 / 2},
$$

where $A, B$, and $C$ are given in (3.5). The eigenvalue equation, obtained by satisfying (4.6), is

$$
W_{1} \operatorname{Im}[\bar{Y}(\nu) Z(\nu)]+W_{2} \operatorname{Im}[\bar{Z}(\nu) X(\nu)]+W_{3} \operatorname{Im}[\bar{X}(\nu) Y(\nu)]=0,
$$

where $\operatorname{Im}(\cdots)$ denotes the imaginary part of $(\cdots)$. The real functions, $W_{1}, W_{2}$, 
and $W_{3}$, are defined as

$$
\begin{aligned}
& W_{1}=-\frac{2 n \nu_{3}^{2} Y_{3}}{\left(\nu_{3} \gamma\right)^{2}}\left\{\nu_{3} \gamma\left[I_{n-1}\left(\nu_{3} \gamma\right)+I_{n+1}\left(\nu_{3} \gamma\right)\right]-2 I_{n}\left(\nu_{3} \gamma\right)\right\}, \\
& W_{2}=2 n \nu_{3} Y_{6} \frac{I_{n}\left(\nu_{3} \gamma\right)}{\nu_{3} \gamma}, \\
& W_{3}=\nu_{3}^{2}\left\{\frac{2 Y_{7}}{\nu_{3} \gamma}\left[I_{n-1}\left(\nu_{3} \gamma\right)+I_{n+1}\left(\nu_{3} \gamma\right)-2 n^{2} \frac{I_{n}\left(\nu_{3} \gamma\right)}{\left(\nu_{3} \gamma\right)}\right]\right. \\
&\left.\quad-Y_{8}\left[I_{n-2}\left(\nu_{3} \gamma\right)+2 I_{n}\left(\nu_{3} \gamma\right)+I_{n+2}\left(\nu_{3} \gamma\right)\right]\right\},
\end{aligned}
$$

with $\gamma=\eta a$; and $Y_{3}, Y_{6}, Y_{7}$, and $Y_{8}$ are given in (3.8). The complex functions $X(\nu), Y(\nu)$, and $Z(\nu)$ are defined as follows:

$$
\begin{aligned}
& X(\nu)=2\left[2 Y_{2}+\nu^{2}\left(2 Y_{1}+Y_{3}\right)\right] J_{n}(\nu \gamma)-\nu^{2} Y_{3}\left[J_{n-2}(\nu \gamma)+J_{n+2}(\nu \gamma)\right], \\
& Y(\nu)=\nu\left(\nu^{2} Y_{4}+Y_{5}\right)\left[J_{n-1}(\nu \gamma)-J_{n+1}(\nu \gamma)\right], \\
& Z(\nu)=2 n Y_{3} \nu^{2}\left\{2 \frac{J_{n}(\nu \gamma)}{(\nu \gamma)^{2}}+\frac{\left[J_{n+1}(\nu \gamma)-J_{n-1}(\nu \gamma)\right]}{\nu \gamma}\right\}
\end{aligned}
$$

where $Y_{1}, Y_{2}, Y_{3}, Y_{4}$, and $Y_{5}$ are, again, given in (3.8).

For the elliptic imaginary subregime, the roots of $\nu_{\alpha}$ are $\pm i p$ and $\pm i q$ and the solution forms for (4.2) are

$$
\varphi=A_{1} I_{n}(\eta p r)+A_{2} I_{n}(\eta q r), \quad \psi=A_{3} I_{n}\left(\eta \nu_{3} r\right)
$$

where $p$ and $q$ are defined as

$$
p^{2}-q^{2}=\left(B^{2}-4 A C\right)^{1 / 2} / A, \quad p^{2}+q^{2}=B / A .
$$

The parameters $A, B$, and $C$ are, again, given in (3.5). The eigenvalue equation for the (EI) subregime is

$$
W_{1}[Q(p) R(q)-Q(q) R(p)]+W_{2}[P(q) R(p)-P(p) R(q)]+W_{3}[P(p) Q(q)-P(q) Q(p)]=0,
$$

where $W_{1}, W_{2}$, and $W_{3}$ have been defined in (4.10). The functions $P(\xi), Q(\xi)$, and $R(\xi)$ are

$$
\begin{aligned}
& P(\xi)=2\left[\left(2 Y_{1}+Y_{3}\right) \xi^{2}-2 Y_{2}\right] I_{n}(\xi \gamma)+\xi^{2} Y_{3}\left[I_{n-2}(\xi \gamma)+I_{n+2}(\xi \gamma)\right], \\
& Q(\xi)=\xi\left(Y_{4} \xi^{2}-Y_{5}\right)\left[I_{n-1}(\xi \gamma)+I_{n+1}(\xi \gamma)\right], \\
& R(\xi)=2 n \xi^{2} Y_{3}\left[\frac{I_{n-1}(\xi \gamma)+I_{n+1}(\xi \gamma)}{\xi \gamma}-2 \frac{I_{n}(\xi \gamma)}{(\xi \gamma)^{2}}\right] .
\end{aligned}
$$

In addition, geometric diffuse modes can be formulated in both the hyperbolic and parabolic regimes, in which shear band bifurcation also becomes possible, as shown by Chau [7, 8]. However, since shear band mode is expected to be the dominant mode of failure and we are motivated by the possibility of diffuse mode triggering localization, only the elliptic diffuse modes (which occur preceding the shear band modes) are emphasized in this study. 
5. Barrelling and buckling modes. Although there is no rigorous proof to the conjecture that a critical aspect ratio $(a / L)_{\mathrm{cr}}$, which separates the barrelling and buckling bifurcations, exists, we attempt in this section to calculate $(a / L)_{\mathrm{cr}}$ for some specific material parameters. The term "barrelling" is used here to represent all the axisymmetric bifurcations $(n=0)$ while "buckling" is used to signify all the antisymmetric ones $(n=1)$. That is, the longitudinal wave number, $m$, defined in (4.1), is not necessarily equal to one. More specifically, for $a / L>(a / L)_{\mathrm{cr}}$ barrelling will be the first geometric bifurcation, while for $a / L<(a / L)_{\text {cr }}$ buckling becomes first available. We assume in this section that $G_{t} / G_{l}$ is close to 1 to exclude the possibilities of bifurcations with $n \geq 2$. As shown in the next section, higher circumferential bifurcation modes $(n \geq 2)$ also become possible under compression if $G_{t} / G_{l}$ is not close to 1 . Setting $n=0$ into (4.9) or (4.14) gives, symbolically,

$$
E_{\text {barr }}(a / L, \sigma, C)=0
$$

as the eigenvalue equation for barrelling for either the EC or the EI subregime. Evidently, this condition is a function of the geometrical ratio $(a / L)$, the Cauchy stress tensor $(\boldsymbol{\sigma})$, and the constitutive tensor $(\mathbf{C})$. Similarly, for $n=1,(4.9)$ or (4.14) provides the following eigenvalue equation,

$$
E_{\text {buck }}(a / L, \sigma, C)=0,
$$

as the buckling criterion for either the EC or the EI subregime. If the constitutive constants are known, we can solve (5.1) and (5.2) simultaneously to yield the bifurcation stress and the critical geometrical ratio $(a / L)_{\mathrm{cr}}$. This procedure has been used by Chau [8] to obtain the range of $(a / L)_{\mathrm{cr}}$ for Rudnicki's model [20]: $1.8<m \pi(a / L)_{\mathrm{cr}}<2.3$. His numerical results also show that $(a / L)_{\mathrm{cr}}$ increases slightly with the values of the instantaneous tangent modulus $\left(E / G_{l}\right)$, the ratio of transverse shear modulus to longitudinal shear modulus $\left(G_{t} / G_{l}\right)$, and the magnitude of the compressive confining stress $\left(-\sigma_{r r} / G_{l}\right)$ at the instant of bifurcation (see Fig. 1 of Chau [8]).

Furthermore, due to the nonlinearity between $\left(\sigma_{z z}-\sigma_{r r}\right) /\left(2 G_{l}\right)$ and $\gamma$ involved in the eigenvalue equations, there may exist more than one root for $(a / L)_{\mathrm{cr}}$ that satisfies both (5.1) and (5.2) simultaneously. Such a situation was observed in Fig. 1 (c) of Chau [8] for decreasing $G_{t} / G_{l}$. Further effects of the decrease of $G_{t} / G_{l}$ on the general eigenmodes will be discussed in the next section.

More generally, this approach can be applied to evaluate the critical geometrical ratio for other materials by specifying (2.1) appropriately. For example, if we assume that rubber is incompressible, pressure-insensitive, isotropic, and highly linear elastic (all the constitutive parameters keep their elastic value), we have, in terms of Rudnicki's model [20], $K \rightarrow \infty, r^{*}=2 \nu=1$, and $G_{t}=G_{l}=G$; thus $E=3 G$. For these particular values, the solution for (5.1) and (5.2) is in the elliptic imaginary subregime, and it is $\sigma_{z z} /\left(2 G_{l}\right)=-0.8482$ and $m \pi(a / L)_{\mathrm{cr}} \approx 26$. Figures 2 and 7 of Beatty [3] suggest that both the observed buckling and barrelling modes appear as the longitudinal mode with $m=2$ (note that $v_{r}$ is proportional to $\cos (m \pi z / L)$ ). Probably, the bifurcation mode with $m=2$ occurs (instead of $m=1$ ) because of the unavoidable end friction. Therefore, for highly linear elastic materials we have 
$(L / a)_{\mathrm{cr}} \approx 0.2417$ for $m=2$; but it appears to be too small comparing to the experimental observations for natural rubber: $4.39 \leq(L / a)_{\mathrm{cr}} \leq 4.62$ (Beatty and Hook [5]); and $3.01<(L / a)_{\mathrm{cr}} \leq 3.88$ (Beatty and Dadras [4]). Details about the evolution of the constitutive parameters under external loads are required before more elaborate comparison can be made. However, we should note that the numerical results of Chau [8] give $2.732<(L / a)_{\mathrm{cr}}<3.49$ for $m=2$; surprisingly, this result is about the same as those values reported by Beatty and Dadras [4] for gray natural rubber. It seems to suggest that Rudnicki's model [20] may be useful in describing the behavior of rubber. Nevertheless, $(L / a)_{\mathrm{cr}}$ appears to be quite sensitive to the constitutive model used.

The analytic forms of the axisymmetric $(n=0)$ and the antisymmetric $(n=1)$ long wavelength limits $(\gamma \rightarrow 0)$ are considered here. For small argument, the Bessel and the modified Bessel functions can be expanded into series form (Abramowitz and Stegun [1]):

$$
J_{n}(x)=\sum_{k=0}^{\infty} \frac{(-1)^{k}(x / 2)^{n+2 k}}{k ! \Gamma(n+k+1)}, \quad I_{n}(x)=\sum_{k=0}^{\infty} \frac{(x / 2)^{n+2 k}}{k ! \Gamma(n+k+1)} .
$$

The axisymmetric long wavelength limit $(\gamma \rightarrow 0$ and $n=0)$ for the eigenvalue equation of the EC subregime can be obtained by substitution of (5.3) into (4.9) as

$$
C Y_{4}\left(2 Y_{1}+Y_{3}\right)+2 Y_{2}\left(A Y_{5}-B Y_{4}\right)=0 \text {. }
$$

Substitution of (3.5) and (3.8) into (5.4) gives two roots:

$$
\sigma_{z z}=\sigma_{z z}^{\max }+O\left(\gamma^{2}\right), \quad \sigma_{z z}=2\left(C_{13}+C_{44}\right)+\sigma_{r r}+O\left(\gamma^{2}\right) .
$$

However, the second of (5.5) gives $s>1$ for positive $C_{13} / C_{44}$; thus, the second solution is in the tensile parabolic regime. Therefore, the only root for the axisymmetric long wavelength limit in the elliptic regime is the first of (5.5). Note that the first term of this solution is equal to the stress at maximum load point given in (2.5). A special case of the first of (5.5) has been obtained previously by Chau [7] for Rudnicki's model [20] although a different method of solution was used. More specifically, instead of the velocity potentials, (3.1), Chau [7] used the method of stress rate potentials.

Substitution of (5.3) into (4.14) again yields (5.4) as the axisymmetric long wavelength limit for the EI subregime. We should also remark, without showing the detail, that (5.4) continues to be valid in the hyperbolic regime as the axisymmetric long wavelength limit.

Similarly, setting $n=1$ and substituting (5.3) into (4.9) gives the antisymmetric long wavelength limit for the EC subregime for small $\gamma$ :

$$
A L_{1}+\frac{\gamma^{2}}{24} L_{2}+O\left(\gamma^{2}\right)=0
$$

where $L_{1}$ and $L_{2}$ depend only on the stress state and constitutive parameters. By setting $\sigma_{r r}=0$ in (5.6), we found that the only root for $L_{1}=0$ is $\sigma_{z z}=0$. This suggests that $\sigma_{z z}$ is of the order $\gamma^{2}$. Using this information and reconsidering the 
root for (5.6), we have

$$
\sigma_{z z}=-\frac{1}{4} E_{\tan } \gamma^{2}+O\left(\gamma^{4}\right)
$$

Note that the first term on the right side of (5.7) equals Euler's buckling formula if the elastic Young's modulus is replaced by the tangent modulus; this result was first remarked by Chau [8] using Rudnicki's model [20].

In general, for nonzero $\sigma_{r r}$, a finite solution (i.e., independent of $\gamma$ ) exists for the antisymmetric long wavelength limit, (5.6) (e.g., see Chau [8]). Due to the nonlinearity of (5.6) in $s$ when $\sigma_{r r} \neq 0$, we are unable to obtain a general explicit form for the bifurcation stress. However, a special form of such a finite antisymmetric solution had been reported by Chau [8] for Rudnicki's model [20] when $K \rightarrow \infty$; and the finite solution of Chau [8] is the same as the corresponding solution obtained by Chau and Rudnicki [9] for plane strain deformations.

A similar expansion for (4.14) gives (5.6) again; therefore, the antisymmetric long wavelength limit continues in the EI subregime. Furthermore, one can also show that (5.6) is valid in the hyperbolic regime.

The physical meaning for the long wavelength limit for $n \geq 2$ is not obvious. Actually, the numerical results (given in Sec. 7) suggest that no long wavelength limit exists for $n \geq 2$.

6. Surface instabilities. Three different short wavelength limits are considered in this section, namely (1) surface wrinkling with short longitudinal wavelength $(\gamma \rightarrow \infty$ with $n$ fixed); (2) surface undulation with short circumferential wavelength $(n \rightarrow \infty$ with $\gamma$ fixed); and (3) surface rumpling with both short longitudinal and circumferential wavelength $(n \rightarrow \infty$ and $\gamma \rightarrow \infty)$. The first case has been considered previously by Chau [7, 8] for the axisymmetric case $(n=0)$ and for the antisymmetric case $(n=1)$ (see also Simpson and Spector [26]). For the second case, only the circumferential wave number approaches infinity; this short wavelength bifurcation can be visualized by the appearance of the Devil's Tower National Monument in Wyoming (e.g., see Shimer [23]). For the third case, both the longitudinal and circumferential wave numbers approach infinity; this type of instability has been observed experimentally and is called "orange-peel" mode (Rittel [18]; Rittel et al. [19]).

6.1. Longitudinal short wavelength limit $(\gamma \rightarrow \infty)$. To consider the short wavelength limit $(\gamma \rightarrow \infty$ with $n$ fixed), we can use the following asymptotic form of $J_{n}(x)$ and $I_{n}(x)$ as $x \rightarrow \infty$ (Abramowitz and Stegun [1]):

$$
J_{n}(x) \approx[2 /(\pi x)]^{1 / 2} \cos (x-n \pi / 2-\pi / 4), \quad I_{n}(x) \approx e^{x} /(2 \pi x)^{1 / 2} .
$$

Note that the asymptotic form of $I_{n}(x)$ is independent of the order as $x \rightarrow \infty$. In addition, for $x \rightarrow \infty$, the following relations:

$$
J_{n-2}(x) \approx J_{n+2}(x) \approx-J_{n}(x), \quad J_{n+1}(x) \approx-J_{n-1}(x),
$$

can be shown by using (6.1). Substitution of (6.1) and (6.2) into (4.9) gives the short longitudinal wavelength mode for the EC subregime

$$
(A C)^{1 / 2}\left[Y_{5}\left(Y_{1}+Y_{3}\right)-Y_{2} Y_{4}\right]+Y_{2} Y_{5} A+Y_{4}\left[C\left(Y_{1}+Y_{3}\right)-Y_{2} B\right]=0 \text {. }
$$


Substitution of (3.5) and (3.8) into (6.3) gives

$$
\begin{array}{r}
{\left[C_{11} C_{13}\left(1-s^{2}\right)\right]^{1 / 2}\left\{\left(C_{31}+2 s C_{44}+\sigma_{r r}\right)\left(C_{13}+\sigma_{r r}\right)-C_{11} C_{33}\right\}} \\
-2 C_{11} C_{33}\left\{(1-s)\left[C_{44} s+\sigma_{r r}\right]-\frac{\sigma_{r r}^{2}}{2 C_{44}}\right\}=0 .
\end{array}
$$

For $\sigma_{r r}=0,(6.4)$ further reduces to

$$
\sigma_{z z}\left[\frac{C_{13}}{C_{11} C_{33}-C_{13} C_{31}}\right]=1+\sigma_{z z}\left[\frac{\left(C_{11} C_{33}\right)^{1 / 2}}{C_{11} C_{33}-C_{13} C_{31}}\right]\left[\frac{2 C_{44}-\sigma_{z z}}{2 C_{44}+\sigma_{z z}}\right]^{1 / 2} .
$$

For an incompressible solid obeying normality subjected to zero confining stress, Chau [7, 8] remarked that $(6.5)$ can be simplified to the bifurcation condition for an incompressible half-space satisfying normality (Biot [6]; Bassani et al. [2]). Actually, (6.4) can be shown analytically to be equal to the condition for surface bifurcation of a half-space regardless of the detail of its constitutive response; however, the proof will not be given here for the sake of conciseness. Employing the framework of hyperelasticity, Simpson and Spector [26] have shown that the reason is that this bifurcation corresponds to failure of the Complementing Condition for the appropriate half-space boundary value problem. This result has also been described and used by Davies [11].

As expected, (6.4) is independent of the circumferential wave number, $n$, because for very short wavelength the curvature of the curved surface plays no role. Nevertheless, this provides an elegant proof of the conjecture that the short wavelength limits for the axisymmetric $(n=0)$ and the antisymmetric $(n=1)$ modes are indeed the same as suspected by Chau [8]. In terms of Rudnicki's model [20], the dependence of the eigenstress for this short wavelength limit on the transverse isotropy, the compressive confining stress, and the in-plane compressibility were examined in detail by Chau [8].

To obtain the same short wavelength limit $(m \rightarrow \infty$, with $a / L$ and $n$ fixed) for the EI subregime, we can substitute (6.1) into (4.14). Again, (6.4) is obtained. Note that similar kinds of longitudinal short wavelength limits have been shown available in both the hyperbolic and the parabolic regimes by Chau [7] for the axisymmetric mode $(n=0)$ and by Chau [8] for the antisymmetric one $(n=1)$.

6.2. Circumferential short wavelength limit $(n \rightarrow \infty)$. The appearance of this kind of surface instability is similar to the parallel wrinkles observed by Rittel [18] for "extruded aluminum 1100" circular cylinders under compression. To obtain the asymptotic form for $J_{n}(x)$ and $I_{n}(x)$ as $n \rightarrow \infty$, we can retain only the first term in (5.3), then apply Stirling's formula for the gamma function for large argument. The result is

$$
I_{n}(x) \approx J_{n}(x) \approx(2 \pi n)^{-1 / 2}[x e /(2 n)]^{n}
$$

if higher-order terms of order $1 / n$ are neglected. Note that all the Bessel functions involved in the eigenvalue equations can be converted to $J_{n}(x), I_{n}(x), J_{n}^{\prime}(x)$, and $I_{n}^{\prime}(x)$ (the superposed prime denotes the first derivative with respect to the argument). 
Following the same procedure used in obtaining (6.6), we find

$$
J_{n}^{\prime}(x) \approx I_{n}^{\prime}(x) \approx(2 \pi n)^{-1 / 2}[x e /(2 n)]^{n}(n / x) .
$$

Substitution of (6.6) and (6.7) into (4.9) leads to the following condition:

$$
Y_{3}-\left(Y_{7}+Y_{8}\right)=0
$$

for the short circumferential wavelength limit for the EC subregime. Similarly, substitution of (6.6) and (6.7) into (4.14) leads again to (6.8) for the EI subregime. Note that the same bifurcation condition is also obtained for both the hyperbolic and the parabolic regimes for this particular limit. However, this condition is satisfied identically regardless of the stress level, that is, this kind of surface instability is always possible if the end surfaces sustain no shear traction. This result seems unable to explain the inception of the parallel wrinkles observed by Rittel [18] under compression.

It is plausible that the parallel wrinkles observed are due to the end friction which promotes bulging and tensile hoop stress. Such tensile hoop stress may eventually lead to the type of surface undulation described in Sec. 6.1. Another possibility is that the wrinkle instability parallel to the cylinder axis is the consequence of slipping that may occur at the end surfaces. When slipping occurs, end friction is released. Thus, the kind of instability considered in this section may be triggered. Furthermore, note that (6.8) is only valid for $n \rightarrow \infty$; however, in reality the wave number (or the wavelength) is likely to be controlled by the grain size of the materials. Thus, for some large but finite $n$ a nontrivial solution different from (6.8) may exist to give a meaningful eigenvalue equation. Nevertheless, further studies, both theoretical and experimental, are required to clarify these possibilities.

6.3. Longitudinal and circumferential short wavelength limit $(n \approx \gamma \rightarrow \infty)$. The "orange-peel" mode of surface instability for circular metal cylinders has been observed by Rittel [18] under compression and by Rittel et al. [19] under tension. We assumed that this "orange-peel" mode corresponds to the case that both the longitudinal and the circumferential wave number become unbounded simultaneously $(\gamma \approx n \rightarrow \infty)$, although, as remarked by Rittel [18], the observed wavelength should actually depend on the grain size. The evaluation of the asymptotic form for $J_{n+1}(n x)$ (similarly for $I_{n+1}(n x), \ldots$ etc.) as $n \rightarrow \infty$ is not straightforward; however, as mentioned in the previous section, we can first rewrite (4.9) and (4.14) in terms of the modified Bessel functions of order $n$ and their derivatives (i.e., $J_{n}(n x), I_{n}(n x), J_{n}^{\prime}(n x)$, and $\left.I_{n}^{\prime}(n x)\right)$. Therefore, the eigenvalue equation for the type of surface undulation in the EC subregime can be obtained by using the following uniform asymptotic forms of $J_{n}(n x)$ and $J_{n}^{\prime}(n x)$ (Abramowitz and Stegun [1]) as $n \rightarrow \infty$ :

$$
\begin{aligned}
& J_{n}(n x) \approx\left[4 \zeta /\left(1-x^{2}\right)\right]^{1 / 4} \mathrm{Ai}\left(n^{2 / 3} \zeta\right) n^{-1 / 3}, \\
& J_{n}^{\prime}(n x) \approx \frac{2}{x}\left[\left(1-x^{2}\right) /(4 \zeta)\right]^{1 / 4} \mathrm{Ai}^{\prime}\left(n^{2 / 3} \zeta\right) n^{-2 / 3},
\end{aligned}
$$

where $\operatorname{Ai}(x)$ and $\operatorname{Ai}^{\prime}(x)$ are the Airy function and its first derivative, respectively; and $(2 / 3) \zeta^{3 / 2}=\ln \left\{\left[1+\left(1-x^{2}\right)^{1 / 2}\right] / x\right\}-\left(1-x^{2}\right)^{1 / 2}$ or, equivalently, $(2 / 3)(-\zeta)^{3 / 2}=$ 
$\left(x^{2}-1\right)^{1 / 2}-\cos ^{-1}(1 / x)$. The variable $\zeta$ should be chosen from either one of these two definitions such that $\zeta$ is real when $x$ is positive. The asymptotic forms of $\mathrm{Ai}(x)$ and $\mathrm{Ai}^{\prime}(x)$ are (Abramowitz and Stegun [1]):

$$
\operatorname{Ai}(x) \approx \frac{1}{2} \pi^{-1 / 2} x^{-1 / 4} \exp \left(-\frac{2}{3} x^{3 / 2}\right), \quad \mathrm{Ai}^{\prime}(x) \approx-\frac{1}{2} \pi^{-1 / 2} x^{1 / 4} \exp \left(-\frac{2}{3} x^{3 / 2}\right)
$$

as $x \rightarrow \infty$. Substitution of $(6.10)$ into (6.9) yields

$$
\begin{aligned}
& J_{n}(n x) \approx \frac{1}{2}\left[4 /\left(1-x^{2}\right)\right]^{1 / 4}(\pi n)^{-1 / 2} \exp \left(-\frac{2}{3} n \zeta^{3 / 2}\right), \\
& J_{n}^{\prime}(n x) \approx \frac{1}{x}\left[\left(1-x^{2}\right) / 4\right]^{1 / 4}(\pi n)^{-1 / 2} \exp \left(-\frac{2}{3} n \zeta^{3 / 2}\right) .
\end{aligned}
$$

The uniform asymptotic expansions for $I_{n}(n x)$ and $I_{n}^{\prime}(n x)$ as $n \rightarrow \infty$ are (Abramowitz and Stegun [1]):

$I_{n}(n x) \approx(2 \pi n)^{-1 / 2}\left(1+x^{2}\right)^{-1 / 4} \exp (n \lambda), \quad I_{n}^{\prime}(n x) \approx(2 \pi n)^{-1 / 2}\left(1+x^{2}\right)^{1 / 4} x^{-1} \exp (n \lambda)$,

where $\lambda=\left(1+x^{2}\right)^{1 / 2}+\ln \left\{x /\left[1+\left(1+x^{2}\right)^{1 / 2}\right]\right\}$. Substitution of (6.11) and (6.12) into (4.9) gives the following condition:

$$
\begin{aligned}
& Y_{3}^{2} Y_{4}\left[2\left(4 A C-B^{2}\right)\left(1+\nu_{3}^{2}\right)\right]^{1 / 2}[(A+B+C) / A]^{1 / 4} \\
& +\left[\left(4 A C-B^{2}\right)(1+D)\right]^{1 / 2}\left\{Y_{3} Y_{6}\left(Y_{1}+Y_{3}\right)\right. \\
& \left.\quad+\left[Y_{8}\left(1+\nu_{3}^{2}\right)+Y_{7}\right]\left[Y_{4}\left(Y_{2}-Y_{3}\right)-Y_{5}\left(Y_{1}+Y_{3}\right)\right]\right\} \\
& +(1-D)^{1 / 2}\left\{Y_{3} Y_{6}\left[2 A\left(Y_{2}-Y_{3}\right)-B\left(Y_{1}+Y_{3}\right)\right]\right. \\
& -\left[Y_{8}\left(1+\nu_{3}^{2}\right)+Y_{7}\right]\left[2 Y_{4} C\left(Y_{1}+Y_{3}\right)-B\left[Y_{5}\left(Y_{1}+Y_{3}\right)+Y_{4}\left(Y_{2}-Y_{3}\right)\right]\right. \\
& \left.\left.+2 Y_{5} A\left(Y_{2}-Y_{3}\right)\right]\right\}=0
\end{aligned}
$$

where $D=(2 A+B) /\left[2 A^{1 / 2}(A+B+C)^{1 / 2}\right]$ for the EC subregime. For the same kind of short wavelength limit in the EI subregime, (6.12) is substituted into (4.14); the resulting eigenvalue equation for the EI subregime is found equal to (6.13) if $\left(4 A C-B^{2}\right)$ and $(1-D)$ are replaced by $\left|4 A C-B^{2}\right|$ and $|1-D|$ respectively.

Numerical results suggest that the root of $(6.13)$ only appears near the tensile elliptic-parabolic $(E / P)$ boundary as $s$ approaches 1 . If we set $(1-s) \rightarrow 0$ for nonzero $\sigma_{r r}$, we find that the first-order term of (6.13) is satisfied identically in the EI subregime. This result can only be applied to explain the observation by Rittel et al. [19] under tension but not the orange-peel mode observed by Rittel [18] for cast aluminum under compression.

The observed orange-peel mode under compression may be the result of the end frictional effect. In particular, if bulging occurs due to the end friction, tensile hoop stress may be developed such that $(6.13)$ is satisfied near the tensile $E / P$ boundary. Further studies including the end frictional effects should give insight to such a possibility.

7. Numerical results for the elliptic diffuse modes. The numerical results for the general eigenvalue equation, (4.9), in the EC subregime are considered in this section. Rudnicki's model [20] is used; $r^{*}$ and $\nu$ are related to the friction coefficient, $\mu$, 


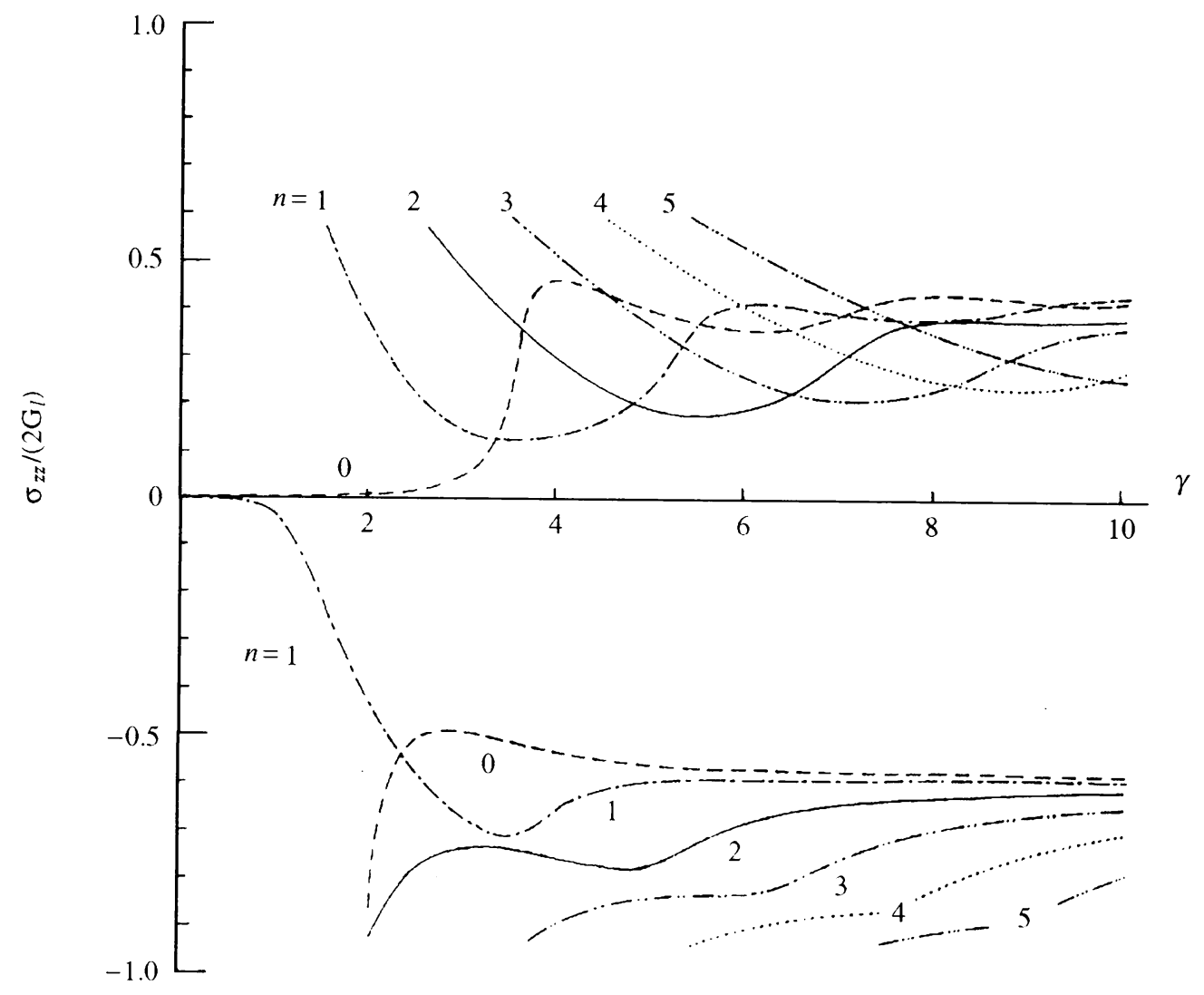

FIG. 1. Eigenvalue surfaces in the elliptic complex subregime for $0 \leq$ $n \leq 5$ with $\mu=\beta=0.4, G_{t} / G_{l}=0.75, G_{l} / K=0.2, \sigma_{r r}=0$, and $E / G_{l}=0$ (at peak stress).

and the dilatancy factor, $\beta$, of the Rudnicki-Rice model [21] as given by (3) of Chau [7]. Only the first six circumferential waves, i.e., $0 \leq n \leq 5$, are evaluated here, although calculations involving higher bifurcation modes $(n>5)$ present no additional difficulty.

Figure 1 is a typical plot to illustrate the eigenvalue surfaces for $n \leq 5$. Under tension $(s>0)$, the lowest possible eigenmode is no longer restricted to either the axisymmetric $(n=0)$ or the antisymmetric $(n=1)$ one as concluded by Chau [8]. Instead, higher circumferential bifurcation modes $(n \geq 2)$ may become the first possible instability as $\gamma$ increases. More specifically, if $a / L$ increases such that $m \pi a / L$ is larger than 5 , the first possible bifurcation mode will be of wave number $n \geq 2$. However, under compression $(s<0)$, the first possible bifurcation remains either the axisymmetric $(n=0)$ or the antisymmetric $(n=1)$ because the bifurcation modes with $n \geq 2$ require a higher compressive stress level. The eigenvalue surfaces, which only combine the lowest eigenstress of modes with $n=0$ and $n=1$, given in Fig. 1 (a-d) of Chau [8] have been recalculated to include bifurcation modes of higher $n$. However, conclusions similar to those of Fig. 1 are arrived at if $G_{t} / G_{l}$ remains close 


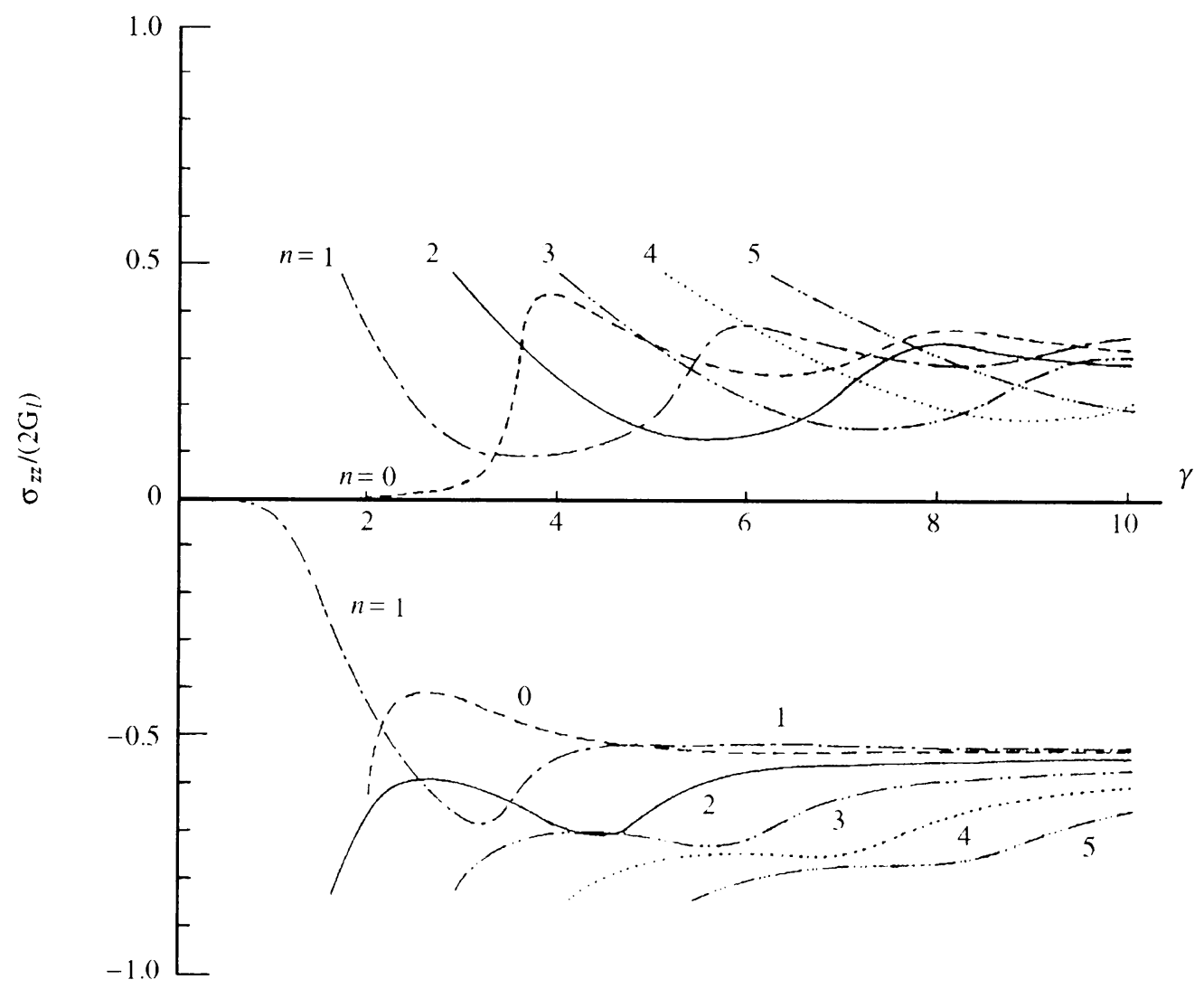

FIG. 2. Same as Fig. 1 with $G_{t} / G_{l}=0.5$.

to 1 . That is, only the tensile eigenvalue envelope for the lowest bifurcation stress is affected; the compressive eigenvalue envelope for the lowest bifurcation stress remains unchanged. Consequently, the new plots including bifurcation modes of higher $n$ will not be given here.

However, if the effect of varying $G_{t} / G_{l}$ is included, the prediction of the lowest possible bifurcation stress under compression is much more complicated. Figure 2 shows an eigenvalue plot similar to Fig. 1 but with smaller value of $G_{t} / G_{l}$ (= $1 / 2)$. Contrary to expectation, the antisymmetric buckling mode $(n=1)$, instead of the axisymmetric bulging $(n=0)$, becomes the first possible bifurcation even for short cylinders (say for $\gamma>5$ ). As $G_{t} / G_{l}$ further decreases, Fig. 3 shows that if $m \pi a / L \approx 2$, the first bifurcation under compression is the eigenmode with $n=2$. In addition, there are five roots for $(a / L)_{\mathrm{cr}}$ for $\gamma \leq 10$; consequently, both buckling and barrelling are equally possible as the first bifurcation depending on the exact value of $m \pi a / L$. A similar eigenvalue plot for $G_{t} / G_{l}=1 / 10$ is given in Fig. 4 (see p. 242). Bifurcation modes with $n=0,1,2,3$ all become possible (for $\gamma \leq 10$ ) depending on the actual dimension ratio $a / L$. Note, however, that all the eigenvalue surfaces with $n \geq 2$ do not intercept at $\gamma=0$; thus, no long wavelength limit $(\gamma \rightarrow 0)$ 


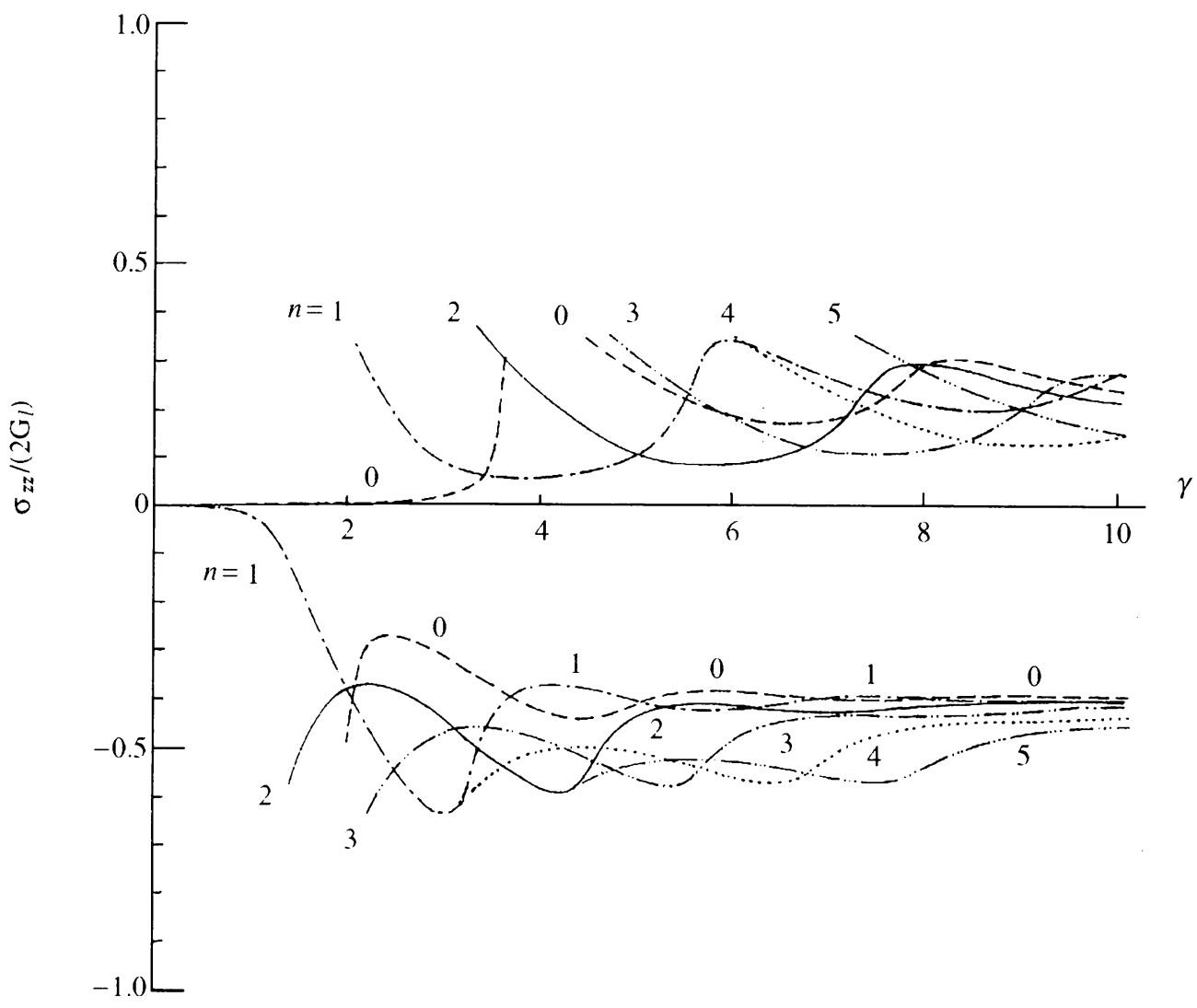

FIG. 3. Same as Fig. 1 with $G_{t} / G_{l}=0.25$.

exists for the circumferential modes with $n \geq 2$.

In short, the ratio of the transverse shear modulus to the longitudinal shear modulus $\left(G_{t} / G_{l}\right)$ appears to be the most crucial factor in determining the earliest possible type of geometric diffuse mode under compression. As discussed by Rudnicki [20] and Chau [7], $G_{t} / G_{l}$ may drop significantly from its elastic value due to the yield vertex effect. Therefore, it is possible that bifurcation modes with $n \geq 2$ may actually become the first bifurcation but, of course, this depends on the current value of $G_{t} / G_{l}$. However, how such higher circumferential diffuse mode bifurcation may trigger pre-peak localization merits further studies.

8. Conclusion. We have considered a general bifurcation analysis for all the geometric diffuse modes for a finite transversely isotropic circular cylinder under initial axisymmetric deformation. This study is a generalization of the previous bifurcation analyses given by Chau $[7,8]$. Both the long wavelength and the short wavelength limits of the general eigenvalue equation are considered. The first term of the axisymmetric long wavelength limit is found equal to the stress at maximum load point; the Euler buckling formula is recovered as the antisymmetric long wavelength limit. In general, a procedure to evaluate the critical geometric ratio, $(a / L)_{\mathrm{cr}}$, that separates 


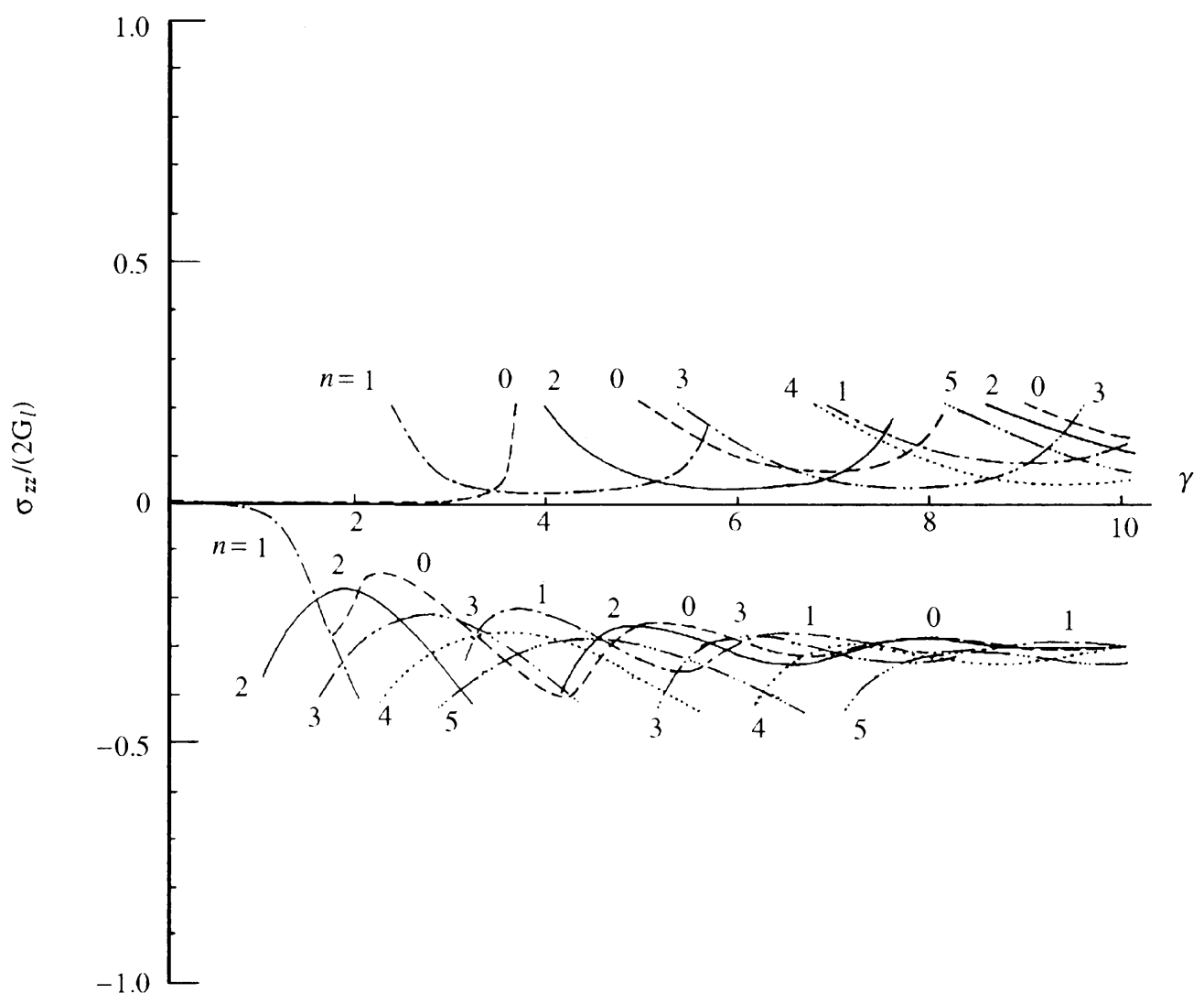

Fig. 4. Same as Fig. 1 with $G_{t} / G_{l}=0.1$.

the buckling mode from the bulging mode, is discussed; numerical results show that $(a / L)_{\mathrm{cr}}$ is very sensitive to the constitutive model used. The bifurcation condition of the longitudinal short wavelength limit ( $\gamma \rightarrow \infty$ with $n$ fixed) is the same as that for a surface wrinkling mode of a half-space; the circumferential short wavelength limit ( $n \rightarrow \infty$ with $\gamma$ fixed) is always a possible bifurcation if no end friction exists; and, finally, the longitudinal and circumferential short wavelength limit $(n \approx \gamma \rightarrow \infty)$, which is assumed corresponding to the "orange-peel" mode observed experimentally, is always satisfied along the tensile $E / P$ boundary.

Neither the circumferential short wavelength limit nor the orange-peel mode short wavelength limit seems able to explain the experimental observations by Rittel [18] under compression if the effect of the end friction is neglected. However, if bulging due to the end friction occurs, tensile hoop stress may develop. Then, if the bifurcation condition for wrinkling instability of half-space is satisfied, parallel wrinkles described by Rittel [18] may appear; if the bifurcation condition for orange-peel mode along the tensile $E / P$ boundary is satisfied, the orange-peel instability observed by Rittel [18] may develop. 
The eigenvalue surfaces including bifurcation modes with $5 \geq n \geq 0$ are evaluated numerically for Rudnicki's model [20] to determine the lowest possible bifurcation stress. If the effect of $G_{t} / G_{l}$ is not significant, the lowest bifurcation mode may be an eigenmode with $n \geq 2$ depending on the actual dimension, $a / L$, under tension; while the lowest bifurcation remains either the axisymmetric $(n=0)$ or the antisymmetric $(n=1)$ mode under compression (in this case $(a / L)_{\text {cr }}$ can be obtained). Conversely, if the ratio of the transverse shear modulus to the longitudinal shear modulus, $G_{t} / G_{l}$, decreases rapidly under deformation, eigenmodes with $n \geq 2$ may also become the first possible bifurcation, again, depending on the cylinder geometry.

Acknowledgments. The work in this study was carried out while the author held a post-doctoral fellowship at Northwestern University. The author is grateful to Professor John W. Rudnicki for helpful discussion. This work was supported by National Science Foundation Grants No. MSM-8612876 and No. MSS-8915607 to Northwestern University.

\section{REFERENCES}

[1] M. Abramowitz and I. A. Stegun (eds.), Handbook of Mathematical Functions, Dover, New York, 1965

[2] J. L. Bassani, D. Durban, and J. W. Hutchinson, Bifurcations at a spherical hole in an infinite. elastoplastic medium, Math. Proc. Camb. Phil. Soc. 87, 339-356 (1980)

[3] M. F. Beatty, Elastic stability of rubber bodies in compression, Finite Elasticity, 27, ASME, AMD, 1977, pp. 125-150 (R. S. Rivlin, ed.)

[4] M. F. Beatty and P. Dadras, Some experiments on the elastic stability of some highly elastic bodies, Internat. J. Engrg. Sci. 14, 233-238 (1976)

[5] M. F. Beatty and D. E. Hook, Some experiments on the stability of circular rubber bars under end thrust, Internat. J. Solids Struct. 4, 623-635 (1968)

[6] M. A. Biot, Mechanics of Incremental Deformations, Wiley, New York, 1965

[7] K. T. Chau, Non-normality and bifurcation in a compressible pressure-sensitive circular cylinder under axisymmetric tension and compression, Internat. J. Solids Struct. 29, 801-824 (1992)

[8] K. T. Chau, Anti-symmetric bifurcations in a compressible pressure-sensitive circular cylinder under axisymmetric tension and compression, ASME J. Appl. Mech. 60, 282-289 (1993)

[9] K. T. Chau and J. W. Rudnicki, Bifurcations of compressible pressure-sensitive materials in plane strain tension and compression, J. Mech. Phys. Solids 38, 875-898 (1990)

[10] P. J. Davies, Buckling and barrelling instabilities in finite elasticity, J. Elast. 21, 147-192 (1989)

[11] P. J. Davies, Buckling and barrelling instabilities of nonlinearly elastic columns, Quart. Appl. Math. 49, 407-426 (1991)

[12] R. Hill, A general theory of uniqueness and stability in elastic-plastic solids, J. Mech. Phys. Solids 6, 236-249 (1958)

[13] R. Hill, Bifurcation and uniqueness in non-linear mechanics of continua, Problems of Continuum Mechanics, contributions in Honor of the Seventieth Birthday of Academician N. I. Muskhelishvili (Ed. by M. A. Lavrent' ${ }^{\prime}$ ev et al.; English edition edited by J. R. M. Radok), Society for Industrial and Applied Mathematics, Philadelphia, PA, 1961, pp. 155-164

[14] R. Hill, Aspects of invariance in solid mechanics, Adv. Appl. Mech. 18, 1-75 (1978) (C. S. Yih, ed.)

[15] M. Levinson, Stability of a compressed neo-Hookean rectangular parallelepiped, J. Mech. Phys. Solids 16, 403-415 (1968)

[16] W. Prager, Introduction to the Mechanics of Continua, Dover, New York, 1973

[17] R. Rice, The localization of plastic deformation, Proc. 14th Internat. Congr. Theoretical and Appl. Mech. (W. T. Koiter, ed.), Delft, North-Holland, Amsterdam, Vol. 1, 1976, pp. 207-220

[18] D. Rittel, The influence of microstructure on the macroscopic patterns of surface instabilities in metals, Scripta Metallurgica et Materialia 24, 1759-1764 (1990)

[19] D. Rittel, R. Aharonov, G. Feigin, and I. Roman, Experimental investigation of surface instabilities in cylindrical tensile metallic specimens, Acta Metall. Mater. 39, 719-724 (1991) 
[20] J. W. Rudnicki, The effect of stress-induced anisotropy on a model of brittle rock failure as localization of deformation, Energy Resources and Excavation Technology, Proc. 18th U. S. Symposium on Rock Mechanics, Keystone, Colorado, June 22-24, 1977, pp. 3B4-1-3B4-8

[21] J. W. Rudnicki and J. R. Rice, Conditions for the localization of pressure-sensitive dilatant materials, J. Mech. Phys. Solids 23, 371-394 (1975)

[22] F. J. Santarelli and E. T. Brown, Failure of three sedimentary rocks in triaxial and hollow cylinder compression tests, Internat. J. Rock Mech. Min. Sci. \& Geomech. Abstr. 26, 401-413 (1989)

[23] J. A. Shimer, This Sculptured Earth: The Landscape of America, Columbia University Press, New York, 1960

[24] H. C. Simpson and S. J. Spector, On barrelling instabilities in finite elasticity, J. Elast. 14, 103-125 (1984)

[25] H. C. Simpson and S. J. Spector, On barrelling for a special material in finite elasticity, Quart. Appl. Math. 42, 99-111 (1984)

[26] H. C. Simpson and S. J. Spector, On the positivity of the second variation in finite elasticity, Arch. Rat. Mech. Anal. 98, 1-30 (1987) 